\title{
Robust conductance estimates from spatial and temporal derivatives of borehole electromagnetic data
}

\author{
Michal Kolaj ${ }^{1}$ and Richard Smith ${ }^{1}$
}

\begin{abstract}
The conductance of an infinite uniformly conductive thin sheet can be calculated using the ratio of the temporal gradient and the spatial gradient in the normal direction of any component (or combination of components) of the secondary magnetic field. With standard borehole electromagnetic (BHEM) systems, the temporal gradient can either be measured or readily calculated from transient-magnetic-field data, and the spatial gradient in the normal direction can be estimated using adjacent stations. Synthetic modeling demonstrates that, for a finite thin sheet, the magnitude of the field provides a robust and reliable apparent conductance in typical three-component BHEM survey configurations. The accuracy in which the apparent conductance can be
\end{abstract}

calculated is hindered by low spatial gradient signal values and can only be reliably estimated where the fields are large (i.e., in close proximity to the target). In a field example of BHEM data collected over a massive sulfide deposit in Sudbury, Ontario, Canada, the spatial gradient could be calculated over a roughly 100m-wide zone, and a consistent apparent conductance could be calculated at each delay time using the magnitude of the field. Increases in the apparent conductance with increasing delay time are likely due to currents migrating into more conductive parts of the body. The apparent conductance values were also consistent with Maxwell models and time constant derived conductance estimates. This simple and robust apparent conductance is ideal as a first-pass estimate for target discrimination, grade estimation, and starting values for forward and/or inversion modeling.

\section{INTRODUCTION}

The success of inductive borehole electromagnetic (BHEM) methods in the exploration for base metals can be attributed to the methods' ability to detect conductive mineralization (which is often the deposit itself) and other conductive geologic features of interest (Dyck, 1991). These conductive features can be identified some distance from the hole (Dyck, 1991). In massive sulfide exploration, the contrast in conductivity between crystalline basement rock and massive sulfides can be as high as $10^{9}$, and with exploration in mature districts such as Sudbury, Ontario, Canada, often exceeding depths of $1 \mathrm{~km}, \mathrm{BHEM}$ is often the primary tool for geophysical exploration and characterization (King, 2007). BHEM interpretation generally involves identifying if there is a conductor proximal to the hole by the existence of an anomalous response. If there is an anomaly, the interpreter will determine the distance to the target (if not intersected) and the orientation of the target. The interpretation exercise is generally undertaken through forward and inversion modeling of the measured data (Dyck, 1991; Polzer, 2000).

The quantitative forward and inversion modeling approach in mineral exploration BHEM varies from manual methods (the interpreter generates synthetic models, using software such as MultiLoop, until the synthetic data resemble the field data; Lamontagne, 2007) to semiautomated methods (the interpreter generates a model that somewhat resembles the field data and then automated inversion fine-tunes the model to provide a better match) to the less common but nearly fully automated methods (inversion algorithms such as the one found in Zhang and Xiao [2001]). In mineral exploration, the targets (especially massive sulfides) can often be well represented as thin sheets and many BHEM interpretation procedures take advantage of this (Grant and West, 1965; Palacky, 1987; Polzer, 2000; King, 2007). Like most of quantitative geophysical techniques, many of these routines are complicated, suffer from nonuniqueness, and the output model may depend strongly on having reliable starting information ( $\mathrm{Li}$ and Oldenburg, 2000;

\footnotetext{
Manuscript received by the Editor 22 October 2013; published online 12 May 2014.

${ }^{1}$ Laurentian University, Department of Earth Sciences, Sudbury, Ontario, Canada. E-mail: mx_kolaj@laurentian.ca; rssmith@laurentian.ca.

(C) 2014 Society of Exploration Geophysicists. All rights reserved.
} 
Lamontagne, 2007; Oldenburg and Pratt, 2007; Lelièvre et al., 2009). It is more convenient to have a simple method to calculate the conductance directly from BHEM data, but typical methods often rely on using circuit theory and/or fitting exponential decays. Historically (and still in practice today), the conductance can be estimated from the decay rate of the body at late time (Nabighian and Macnae, 1991). For a thin sheet, the conductance is proportional to $10 \tau / \mu L$, where $\tau$ is the time constant of the body and $L$ is typically the smallest dimension of the sheet (either the strike or down-dip length). The time constant is generally estimated by fitting an exponential decay to the late time measured response. This method is often only reliable far from the source or at a late time in which the high-frequency information has decayed as the method effectively assumes that only the lowest order decay mode (large and smooth current system) is being observed (B. Polzer, personal communication, 2013).

Three benefits of having reliable estimates of conductivity or conductance (product of conductivity and thickness) are (1) to have better starting models for forward and inversion modeling, (2) for improved target discrimination (King, 2007), and (3) an ability to estimate grade variation within the target (McDowell et al., 2007).

An independent way of obtaining a conductivity/conductance estimate is to use a galvanic or inductive borehole probe or laboratory equipment (e.g., handheld conductivity meter on the core, Smith et al., 2012). In addition to having to collect additional data, different borehole probes and laboratory techniques measure the conductivity over a different scale length and typically often provide drastically different values. Other difficulties with these measurements include the availability of equipment, the additional cost, and the fact that the samples may not be available (i.e., no recovered core; Smith et al., 2012). As such, the use of a simple and robust method to calculate the target conductance directly from BHEM data is justified.

The method presented in this paper uses the formulation of Kolaj and Smith (2013), who develop a methodology to estimate the conductance of a thin sheet from time-domain EM data using the ratio of the temporal gradient and the gradient in the vertical direction of the vertical secondary magnetic field. This work was done assuming that the buried sheet was horizontal and that the receivers were on the surface. We show that, for an infinite uniform sheet, any magnetic-field component (or combination of components) can be used to estimate the conductance. Through synthetic studies, we show that reliable results can be obtained when using the magnitude of the magnetic field to calculate an apparent conductance for finite-size sheets. This apparent conductance is robust because it is relatively insensitive to varying transmitter positions, the borehole location within the target, and the borehole/sheet orientation. Last, we show results from a field example, where the apparent conductance is calculated from two typical three-component BHEM surveys collected over a massive sulfide target in Sudbury, Ontario, Canada.

\section{THEORY}

The thin-sheet assumption allows for substantial simplification of the EM induction problem due to the ability to define a surface current that is constrained to flow only in the plane of the sheet (Price, 1949). Furthermore, simple relationships between the magnetic-field components directly above and below the sheet can be derived (Grant and West, 1965). Following Price (1949),
Smith and West (1987), and Kolaj and Smith (2013), these simplifications allow for a simple differential equation for a flat-lying thin sheet in a resistive medium to be derived as

$$
-\frac{d H_{z}^{s}}{d z} R+\frac{d R}{d y} H_{y}^{s}+\frac{d R}{d x} H_{x}^{s}=-\frac{\mu}{2} \frac{d H_{z}}{d t},
$$

where $R(x, y)$ is the resistance of the sheet; $\mu$ is the magnetic permeability (generally assumed to be that of free space); and the variables $d H_{z}^{s} / d z, H_{y}^{s}, H_{x}^{s}$, and $d H_{z} / d t$ are measured directly above (or below) the sheet, where the vector field $\mathbf{H}^{S}=\left(H_{x}^{s}, H_{y}^{s}, H_{z}^{s}\right)$ represents the secondary field emanating from the sheet and $\mathbf{H}$ represents the vector of the total field (secondary field plus the primary field from the transmitter, $\mathbf{H}=\mathbf{H}^{S}+\mathbf{H}^{P}$ ). In equation 1, the sheet is assumed to be in the horizontal plane, which, if untrue, the three-component magnetic field $\left(H_{z}, H_{x}, H_{y}\right)$ would need to be rotated so that the $H_{z}$-component was normal to the sheet. As discussed in Kolaj and Smith (2013), by assuming that the sheet is infinite in extent and that the sheet is of uniform resistance so the $d R / d x$ and $d R / d y$ terms can be ignored, a conductance can be estimated from

$$
C=\frac{2}{\mu}\left(\frac{\frac{d H_{n}^{s}}{d n}}{\frac{d H_{n}}{d t}}\right)
$$

where $C$ is the conductance (inverse of the resistance $R$ ) and $n$ represents the normal component to the thin sheet such that the restriction that the sheet is in the horizontal plane is no longer necessary. Furthermore, in the off time or when the primary field is constant, the temporal derivative will be solely a secondary field (i.e., $\left.d H_{n}^{P} / d t=0\right)$. This ratio can therefore be independent of the transmitter (location and current waveform), which adds to the simplicity of the method. If the sheet is not uniform or infinite, then equation 2 will not yield a conductance, but an apparent conductance.

Equation 2 can be investigated in more detail using image theory in cylindrical coordinates (Grant and West, 1965). If the transmitting source is a vertical dipole situated at $(0, h)$ with moment $m$ and the infinite sheet of conductance $C$ is located in the plane $z=0$, then the secondary magnetic fields can be shown to be equal to

$$
\mathbf{H}^{S}(\rho, z, t)=\frac{m}{4 \pi}\left[\frac{3 \rho a}{\left(\rho^{2}+a^{2}\right)^{\frac{5}{2}}} \mathbf{i}_{p}+\frac{2 a^{2}-\rho^{2}}{\left(\rho^{2}+a^{2}\right)^{\frac{5}{2}}} \mathbf{i}_{z}\right],
$$

where $a=z+h+(2 t / \mu C))$ and $\mathbf{i}_{\rho}$ and $\mathbf{i}_{z}$ are the unit vectors along the $\rho$ and $z$ axes, respectively (there is no field in the $\varphi$-direction because the primary field has no component in this direction; see Telford et al., 1990). This is known as Maxwell's receding image solution (Grant and West, 1965) because the secondary magnetic fields are equal to the image of the source receding downward with a velocity of $(2 t / \mu C)$. Differentiating $\mathbf{H}^{S}$ with respect to $z$ and $t$ yields

$$
\frac{d \mathbf{H}^{S}}{d z}(\rho, z, t)=\frac{3 m}{4 \pi}\left[\frac{\rho\left(\rho^{2}-4 a^{2}\right)}{\left(\rho^{2}+a^{2}\right)^{\frac{7}{2}}} \mathbf{i}_{p}+\frac{a\left(3 \rho^{2}-2 a^{2}\right)}{\left(\rho+a^{2}\right)^{\frac{7}{2}}} \mathbf{i}_{z}\right]
$$




$$
\frac{d \mathbf{H}^{S}}{d t}(\rho, z, t)=\frac{3 m}{2 \pi C \mu}\left[\frac{\rho\left(\rho^{2}-4 a^{2}\right)}{\left(\rho^{2}+a^{2}\right)^{\frac{7}{2}}} \mathbf{i}_{p}+\frac{a\left(3 \rho^{2}-2 a^{2}\right)}{\left(\rho^{2}+a^{2}\right)^{\frac{7}{2}}} \mathbf{i}_{z}\right],
$$

respectively. Notice that the terms on the right side in the square brackets are identical. Thus, by dividing the vertical components of equations 4 and 5 , a factor of $(\mu C / 2)$ remains, which is the expected result based upon equation 2 . However, as is evident in equations 4 and 5, this relationship is also true for other components of the vector, specifically, $H_{\rho}$. This same conclusion can also be deduced by the observation that $H_{\rho}$ and $H_{z}$ are of the same form (equation 3 ) and the difference between the $t$ and $z$ derivative is controlled by the term, $a$, where $d a / d z=0$ and $d a / d t=2 / \mu C$. Because all the components result in the same answer, any linear combination of the components, including the magnitude $H_{m}=$ $\left(H_{\rho}^{2}+H_{z}^{2}\right)^{0.5}$, can be used in the calculation of the conductance. Furthermore, although equation 1 is valid only directly above or below the sheet, equation 2 (with any component) is valid for measurements made at any distance away from the sheet.

Equation 2 requires measurements of the magnetic field and its time derivative, which can be obtained by using a magnetic-field sensor and then estimating its time derivative using adjacent delay times, i.e., a finite forward difference scheme such as

$$
\frac{d \mathbf{H}^{s}\left(k, t_{j}+0.5 \Delta t\right)}{d t}=\frac{\mathbf{H}^{s}\left(k, t_{j+1}\right)-\mathbf{H}^{s}\left(k, t_{j}\right)}{\Delta t},
$$

where $k$ represents the station, $j$ represents the channel number, and $\Delta t$ is the difference in time between the two adjacent time channels. Alternatively, if using an induction coil (time derivative) sensor, then the full-waveform time-derivative measurements can be integrated to give a magnetic field (Smith and Annan, 2000). The derivative in the normal direction of the magnetic field for a flat lying sheet and a vertical borehole can be approximated using the difference between stations, i.e., a finite central difference scheme such as

$$
\frac{d \mathbf{H}^{s}\left(k, t_{j}\right)}{d z}=\frac{\mathbf{H}^{s}\left(k+1, t_{j}\right)-\mathbf{H}^{s}\left(k-1, t_{j}\right)}{\Delta z},
$$

where $z$ is the depth and $\Delta z$ is the change in depth between the station directly below and above station $k$. A comparison of the spatial and temporal derivatives (calculated using equations 6 and 7) and the calculated conductance (equation 2) using different components for an analytical solution of a $1000 \mathrm{~S}$ infinite horizontal sheet at a depth of $300 \mathrm{~m}$ can be seen in Figure 1. As opposed to equations $3-5$, the transmitter is a 400 by $200 \mathrm{~m}$ rectangular loop and is located $100 \mathrm{~m}$ to the east of the borehole. Note that the calculated conductance differs slightly in close proximity to the sheet because the derivatives have been estimated using a finite-difference approach (equations 6 and 7).

Although it is now clear that any component that is not null coupled to the secondary field can be used to calculate the conductance of an infinite sheet, it is not as easy to prove how effective the different components will be at calculating an apparent conductance of finite nonuniform thin sheets. Furthermore, $d \mathbf{H}^{s} / d z=d \mathbf{H}^{s} / d n$ only when the borehole axis is normal to the sheet, and as such, erratic/erroneous conductance estimates may occur when $d \mathbf{H}^{s} / d z$ (equation 7) is used instead of $d \mathbf{H}^{s} / d n$ to calculate the conductance (equation 2) for different survey/model geometries. These issues will be further investigated through forward modeling of finite and dipping thin sheets in the following section.

\section{FORWARD MODELING}

In each example, a $1 \mathrm{~Hz}$ BHEM UTEM survey (West et al., 1984) with station spacing of $10 \mathrm{~m}$ was simulated in MultiLoop III (Lamontagne Geophysics, Walker and Lamontagne, 2006). A general schematic of the synthetic model can be seen in Figure 2. Because a UTEM survey measures the magnetic field at each station, the spatial derivative was calculated using a central difference between stations (equation 7) and the time derivative was calculated using the forward difference between adjacent delay times (equation 6). Because the time derivative is calculated using adjacent delay times, the spatial derivative is also averaged over adjacent delay times. Additional survey parameters are stated before each example. Note that no noise (apart from some numerical noise) was added to the data.

a)

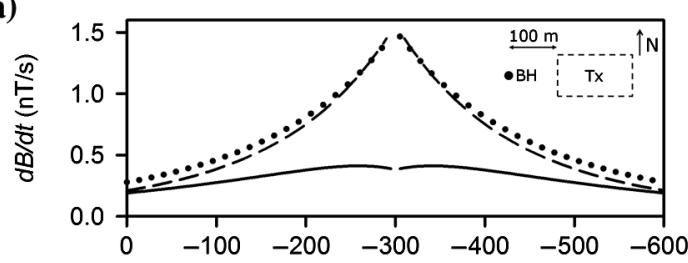

b)
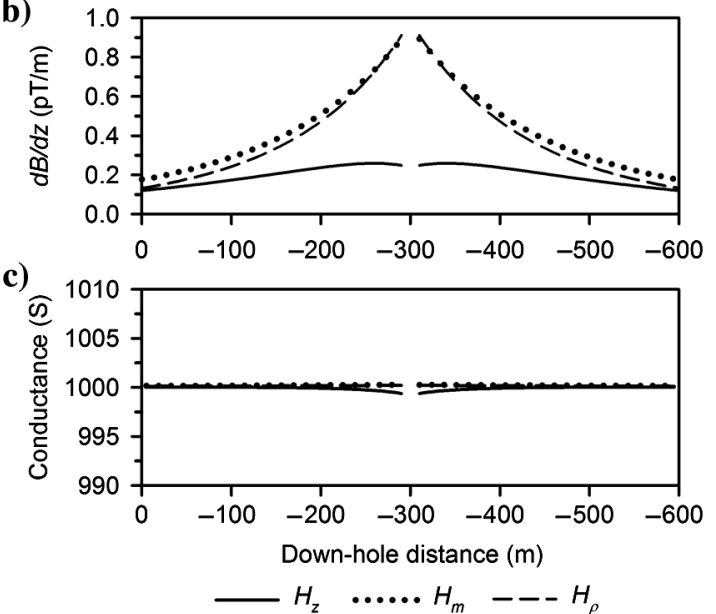

Figure 1. Comparison of equation 2 using $H_{z}$ (solid line), $H_{m}$ (dotted line), and $H_{\rho}$ (dashed line) for a step response at $t=1.1 \mathrm{~ms}$ intersecting a horizontal $1000 \mathrm{~S}$ infinite sheet located at a depth of $-300 \mathrm{~m}$. The station spacing is $5 \mathrm{~m}$, and several stations in close proximity to the sheet have been removed. A $400 \times 200 \mathrm{~m}$ transmitter is located $300 \mathrm{~m}$ east of the borehole (geometry is depicted in the top right corner). (a) Absolute magnitude of the time derivative of the vertical component $\left(d B_{z} / d t\right)$, the magnitude $\left(d B_{m} / d t\right)$, and the horizontal component $\left(d B_{\rho} / d t\right)$. (b) Absolute magnitude of the spatial derivative of the vertical component $\left(d B_{z}^{S} / d z\right)$, the magnitude $\left(d B_{m}^{S} / d z\right)$, and the horizontal component $\left(d B_{\rho}^{S} / d z\right)$. (c) Conductance calculated from the ratio of the fields in (a) and (b). Note that $\mathbf{B}$ magnetic fields are shown in this and subsequent figures because $\mathbf{B}$-field units (nT) are more commonly used in practice (conversion $\mathbf{B}=\mu \mathbf{H}$ ). 


\section{Synthetic example 1 - Zero dip}

In the first example, a $600 \times 600 \mathrm{~m}$ loop was positioned around the survey area (Figure 2) containing a $250 \times 250 \mathrm{~m}$ sheet at a depth of $200 \mathrm{~m}$ having a conductance of $10,000 \mathrm{~S}$ with boreholes $(\mathrm{BH})$ oriented normal to the sheet (i.e., no dip; $d \mathbf{H}^{s} / d z=d \mathbf{H}^{s} / d n$ ). The results of equation 2 for the early and late times using the vertical $\left(H_{n}=H_{z}\right)$, horizontal $\left(H_{\rho}\right)$, and magnitude $\left(H_{m}\right)$ components for BH\#41 (central hole) and BH\#21 (corner of sheet) can be seen in Figure 3 (there is no horizontal component for the central borehole because these fields are essentially null coupled to the body).

For the central borehole (BH\#41, top two panels, Figure 3), the apparent conductance calculated using the vertical and magnitude components is the same because the magnitude of the field is equal to the vertical field $\left(H_{z} \gg H_{\rho}\right.$ thus $\left.H_{m} \approx H_{z}\right)$ in this symmetric central-loop configuration (Figure 2). In both cases, close to the sheet $(-180$ or $-220 \mathrm{~m})$, an apparent conductance of 9000 to $10,000 \mathrm{~S}$ could be estimated depending on which delay time is used. For the borehole intersecting the top left of the sheet (BH\#21, bottom two panels, Figure 2), the apparent conductance using different components differs. The vertical component produces erroneous results at the late delay time where the conductance drops off quite rapidly away from the sheet. Nevertheless, an apparent conductance of 6500 to $7500 \mathrm{~S}$ could be estimated. Overall, the apparent conductance from $H_{\rho}$ is larger and the apparent conductance calculated using $H_{\rho}$ and $H_{m}$ drops off steadily away from the sheet. A reliable apparent conductance of 7500 to $10,000 \mathrm{~S}$ could be estimated from either of the two methods. If the methods' assumptions are true (infinite uniform sheet), then all time channels should produce the same calculated conductance and the apparent conductance will be equal to the actual target conductance. However, because the

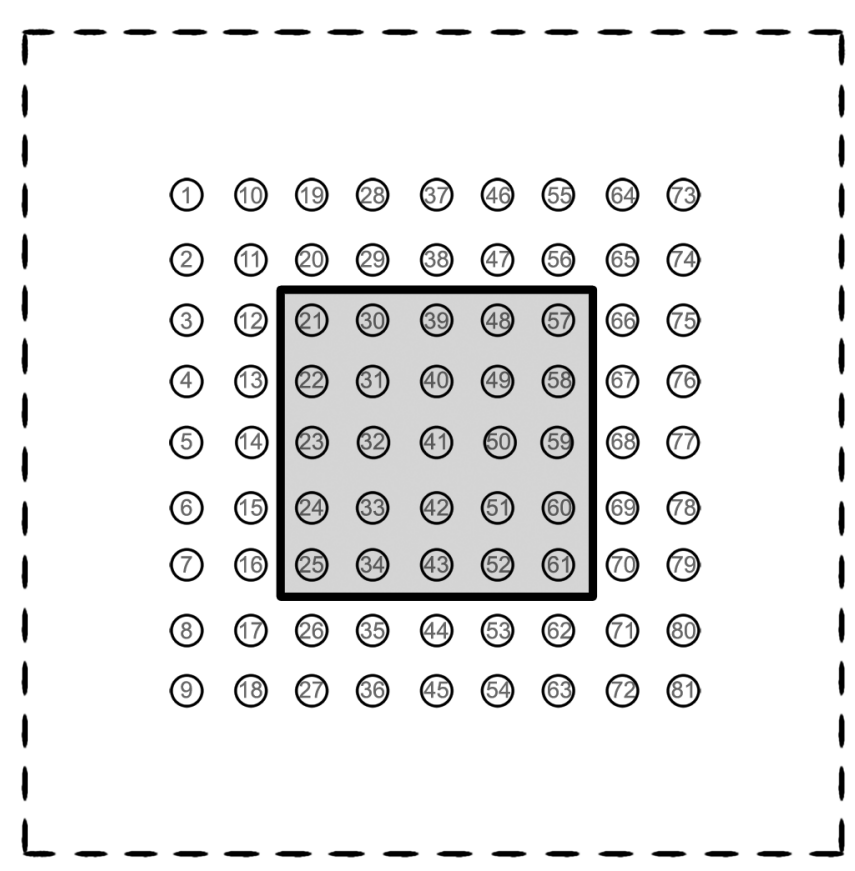

Figure 2. Plan view of the generalized survey geometry for the synthetic models used in MultiLoop III. The dashed black line represents the transmitter loop, circles represent the boreholes (numbered $1-81$, spaced $50 \mathrm{~m}$ apart), and the central gray square represents the surface projection of the thin sheet. sheet is finite in size and each time channel corresponds to a current system with a different shape, discrepancies in the calculated apparent conductance can arise because the methods' assumptions are violated to a varying extent. Furthermore, differences can also arise from errors in the finite-difference approximation used to calculate the temporal and spatial derivatives. In this example, the later delay times produced higher conductance values.

The later delay times produced results closer to the actual conductance, with channel $2(t=140.6 \mathrm{~ms})$ providing a good estimate. A gridded map of the apparent conductance from all boreholes at channel 2 at a depth of $180 \mathrm{~m}$ can be seen in Figure 4. For the vertical component $\left(H_{z}\right)$, there seemed to be an edge effect for stations in close proximity to the sheet edge at a depth of $180 \mathrm{~m}$, so for these holes, the estimate from a depth of $190 \mathrm{~m}$ was used. Similarly, for the horizontal component $\left(H_{\rho}\right)$, there were erroneously high apparent conductance values for the boreholes that did not intersect the sheet and a depth of $160 \mathrm{~m}$ for these holes resulted in improved results. These issues were not seen in the estimates obtained using the magnitude $\left(H_{m}\right)$, suggesting this may be a more robust method.
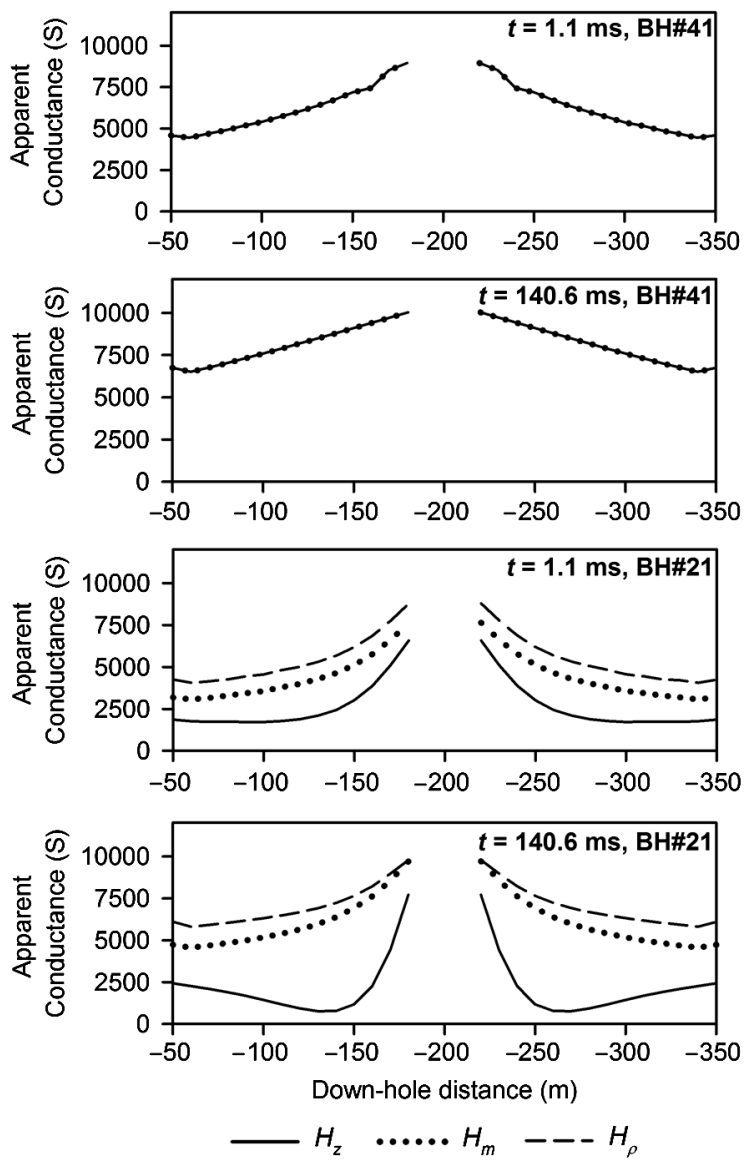

Figure 3. Apparent conductance calculated using equation 2 using $H_{z}$ (solid line), $H_{m}$ (dotted line), and $H_{\rho}$ (dashed line) at the early and late times for the synthetic model shown in Figure 2. The sheet is located at $-200 \mathrm{~m}$, the conductance of the sheet is $10,000 \mathrm{~S}$, and $\mathrm{BH} \# 41$ and $\mathrm{BH} \# 21$ are the boreholes going through the center and top left corner of the sheet, respectively (Figure 2). Time 0 corresponds to the moment of slope change in the UTEM triangle current waveform. The time windows correspond to the mean middle time of the time windows used. Stations 190 to $210 \mathrm{~m}$ downhole have been removed due to the high numerical noise close to the sheet. 
In Figure $4, H_{z}$ produces a relatively diffuse anomaly and the apparent conductance drops off steadily away from the sheet center. The image from $H_{\rho}$ is less diffuse, but it overestimates the actual conductance over most of the sheet and contains some edge effects for the nonintersecting boreholes. The $H_{m}$ image has all of the sought characteristics: high resolution, minimal boundary effects, and a consistent apparent conductance estimate over most of the sheet. Although each method would likely provide an apparent conductance estimate of about the same order of magnitude, $H_{m}$ appears to be superior.

\section{Synthetic examples $2 \& 3-$ Dip of $45^{\circ}$}

In the second and third examples, the $10,000 \mathrm{~S}$ sheet is given a $\operatorname{dip}$ of $45^{\circ}$ (i.e., the boreholes are no longer normal to the sheet). The sheet's dimensions were changed to $350 \times 250 \mathrm{~m}$ so that the number of boreholes intersecting the sheet remains the same as in example 1. In example 3, the effect of the transmitter location is investigated by offsetting the transmitter loop $700 \mathrm{~m}$ to the east such that none of the boreholes are inside of the loop. Because the boreholes are not normal to the sheet in either example 2 or 3 , the spatial gradient (equation 7) is no longer calculated along the normal direction but rather along the borehole axes (vertical in this case; $d \mathbf{H}^{s} / d z$ is now only an approximation of $\left.d \mathbf{H}^{s} / d n\right)$. This approximation should hold as long as the distance between stations is small and the secondary magnetic fields are not rapidly varying over that
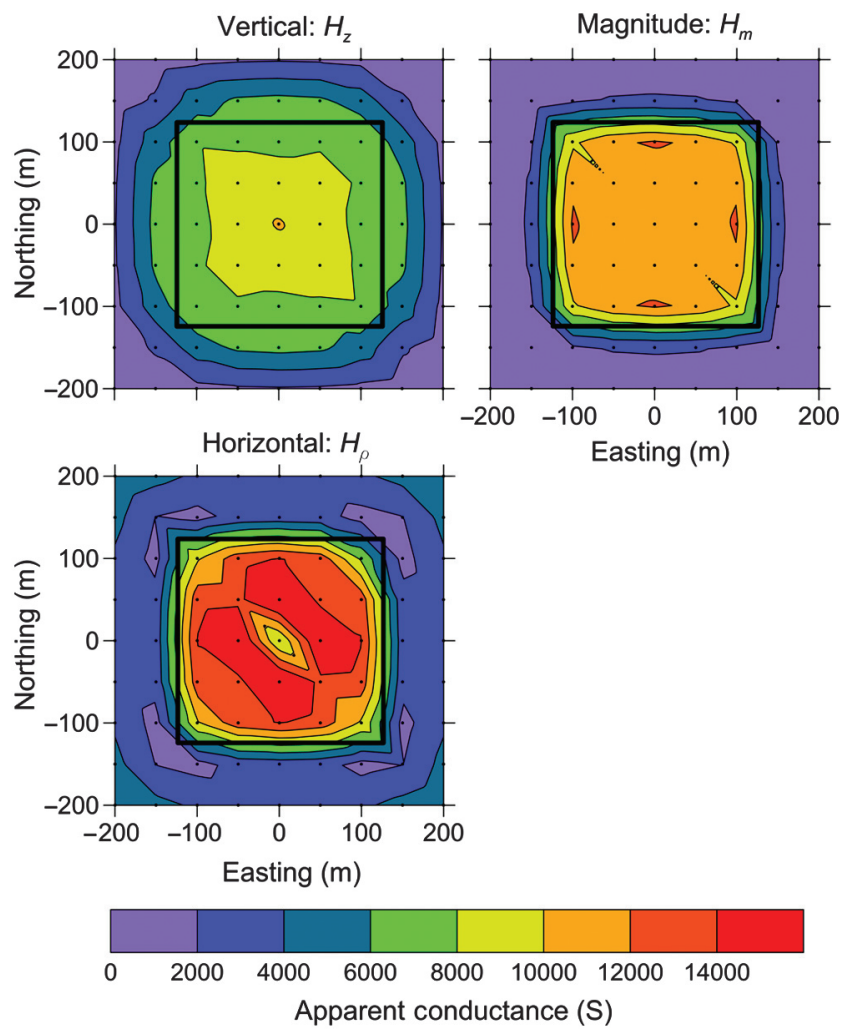

Figure 4. Gridded apparent conductance at the late time $(t=$ $140.6 \mathrm{~ms}$ ) using $H_{z}$ (top left), $H_{m}$ (top right), and $H_{\rho}$ (bottom left) corresponding to the survey layout seen in Figure 2 . The black dots represent borehole locations, and the thick black line is the outline of the $10,000 \mathrm{~S}$ sheet. The image is generated from estimates at a depth of approximately $180 \mathrm{~m}$ (see text). small distance. Because the magnitude was the most successful component in the previous example, the calculated apparent conductance using the magnitude on examples 2 and 3 for BH\#41 and BH\#21 (the central and corner holes, respectively) can be seen in Figure 5.

In contrast to the first example, the magnetic field down the borehole is no longer symmetric about the plane of the sheet due to the nonzero dip. As such, the calculated apparent conductance is also often not symmetric about the sheet, and in some cases, the apparent conductance values and range is smaller on one side of the sheet. Large asymmetry in the apparent conductance generally results from discontinuities in either gradient and/or from differences in decay rates above and below the sheet. As such, there is some ambiguity as to which apparent conductance values to use, but because they tend to be of the same order of magnitude, an average of both sides tended to produce reasonable estimates. As before, the later delay times produce higher apparent conductance estimates. Although equation 2 for the infinite sheet is independent of the transmitter properties, it is evident that there is some effect on the apparent conductance when the transmitter is offset from a finite sheet (i.e., compare Tx_1 and Tx_2 in Figure 5). This difference is

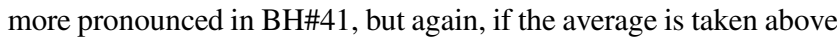
and below the sheet, both transmitters produce roughly the same apparent conductance. Using the average above and below, an apparent conductance of roughly 6000-9000 S could be estimated, which is close to the actual conductance of $10,000 \mathrm{~S}$. As before, all boreholes can be used to produce a contour map of the apparent conductance. A gridded apparent conductance map (channel 2, $t=140.6 \mathrm{~ms}$ ) was calculated using the average apparent conductance of the station $20 \mathrm{~m}$ above and the station $20 \mathrm{~m}$ below the maximum $H_{m}$ value (Figure 6). The maximum $H_{m}$ value was used because this was generally proximal to the location of the sheet (where reliable estimates can be made).
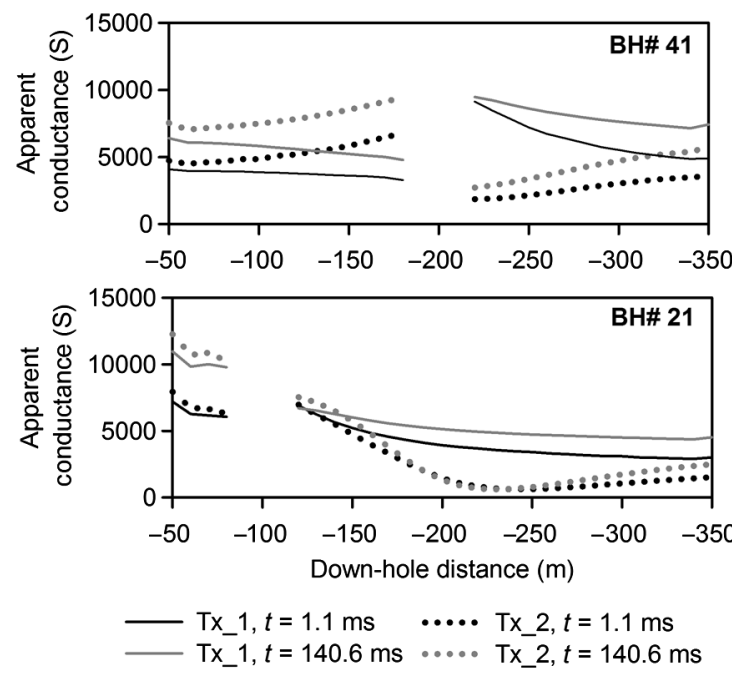

Figure 5. Apparent conductance calculated using equation 2 using $H_{m}$ at an early and late time for the synthetic model shown in Figure 2 (sheet dipping at $45^{\circ}$ ). In contrast to example 1 , the $10,000 \mathrm{~S}$ sheet has dimensions of $350 \times 250 \mathrm{~m}$ and has a dip of $45^{\circ}$. Tx_1 (example 2) represents the survey being performed inside of the transmitter loop (Figure 2), and the transmitter for Tx_2 (example 3 ) has been offset $700 \mathrm{~m}$ to the east. Stations in close proximity to the sheet have been removed. 
The results from Figure 6 are very similar to that of the apparent conductance calculated using $H_{m}$ in Figure 4. There is a welldefined sheet edge, minimal edge effects, and a relatively consistent apparent conductance over the sheet itself. Both transmitter positions essentially produce the same interpreted conductance, showing that for a finite sheet, the apparent conductance is only weakly dependent of the transmitter position. The apparent conductance $(5000-10,000 \mathrm{~S})$ is close to the true conductance $(10,000 \mathrm{~S})$ but is overall a poorer estimate than when the sheet had a zero dip (Figure 4). This is likely due to the fact that the derivative is not the derivative in the normal direction, but a derivative in a direction at $45^{\circ}$ (i.e., $d H_{m}^{s} / d z \neq d H_{m}^{s} / d n$ ). A reduction by a factor of $1 / \sqrt{2}$ would be expected. The apparent conductance was also calculated using other components $\left(H_{z}, H_{\rho}\right.$, rotated $\left.H_{n}\right)$, and the results were considerably erratic (not shown). Some boreholes would produce apparent conductances close to the true value, whereas others were considerably high or low. Overall, none of them produced results as reliable as using $H_{m}$.

\section{Summary and discussion of synthetic modeling}

From the three synthetic examples, it is clear that, although the theory is developed over infinite uniform sheets, the apparent conductance calculated over finite-size sheets is quite reliable and close to the true conductance when the magnitude $\left(H_{m}\right)$ is used. Using $H_{m}$ provides the most robust solution because the apparent conductance is reliable regardless of the transmitter position, the location of the borehole within the target, and the orientation of the borehole and/or the sheet (i.e., even when $d H_{m}^{s} / d z \neq d H_{m}^{s} / d n$ ). Other components produce reliable results intermittently. It should also be mentioned that with real survey data, it would likely not be possible to calculate the apparent conductance down the entire hole as depicted in Figures 3 and 5 due to low signal levels in the spatial gradient $(d \mathbf{H} / d z)$. In reality, an adequate signal-to-noise ratio $(\mathrm{S} / \mathrm{N})$ and thus calculated apparent conductance may only be possible where the fields are large (as would be the case in close proximity

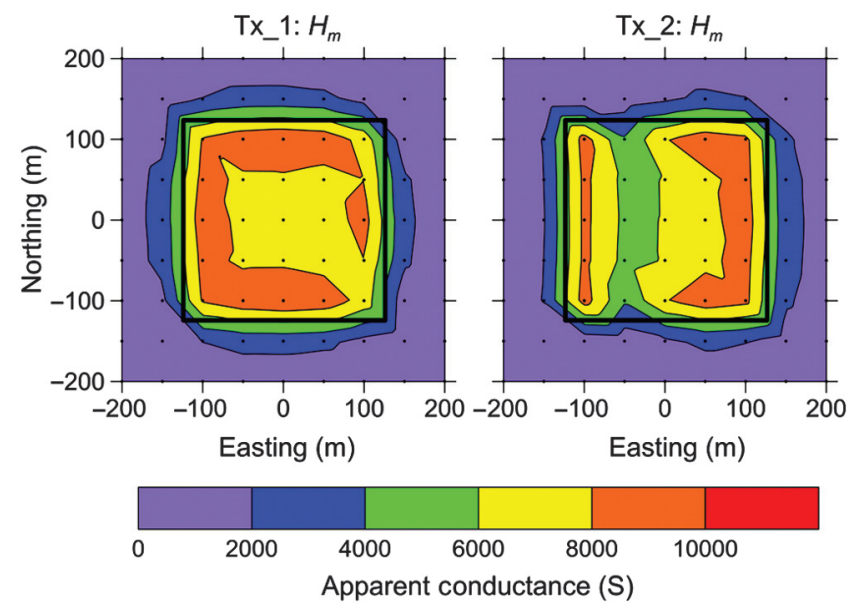

Figure 6. Gridded apparent conductance at the late time $(t=140.6 \mathrm{~ms})$ using the magnitude $\left(H_{m}\right)$ for a $10,000 \mathrm{~S}$ sheet with a dip of $45^{\circ}$. For Tx_1 (left panel), the transmitter encompasses the survey area (Figure 2), and for Tx_2 (right panel), the transmitter has been offset by $700 \mathrm{~m}$ to the east. The conductance values are the average of the conductance estimate $20 \mathrm{~m}$ above and below where the magnetic field is the largest. to the target). Therefore, using the magnitude component has the added benefit that the magnitude of the field will be the largest possible combination of the components and will thus produce the best possible S/N.

\section{FIELD EXAMPLE}

The presented methodology for estimating the target conductance can be readily applied on existing borehole data because the method only requires three-component data, which is generally the standard in modern BHEM. As such, two previously surveyed boreholes (4 Hz UTEM survey with a station spacing ranging from 10 to $15 \mathrm{~m}$, Figure 7) in Sudbury, Ontario, Canada, are used in this study. The massive sulfide deposits in Sudbury were chosen as ideal targets because they can generally be well approximated by one or more thin sheets/plates, which is consistent with the assumptions of the method. The results of equation 2 using $H_{m}$ can be seen in Figures 8 and 9.

As before, the magnitude of the field (Figures 8a and 9a) was calculated from the three-component data, and it is clear that, apart from a roughly $100-\mathrm{m}$-wide zone, the fields are very small over most of the hole. The spatial gradient (equation 7) was calculated using the distance downhole $\left(z_{m}\right)$ rather than the change in depth $(z)$. Borehole\#2 is essentially vertical, and as such, the difference is negligible, whereas for $\mathrm{BH} \# 1$, using the downhole distance will result in conductance values roughly $10 \%-20 \%$ smaller than using the change in depth. Without knowledge of the strike and dip of the target, it is uncertain which strategy will give a derivative closest to the derivative normal to the plate. The derivatives $\left(d B_{m} / d t\right.$ and $d B_{m} / d z_{m}$, Figures $8 \mathrm{~b}$ and $9 \mathrm{~b}$ and Figure $9 \mathrm{~b}$ and $9 \mathrm{c}$, respectively) were calculated using the same finite-difference scheme as in the synthetic study. Because spatial gradients have very

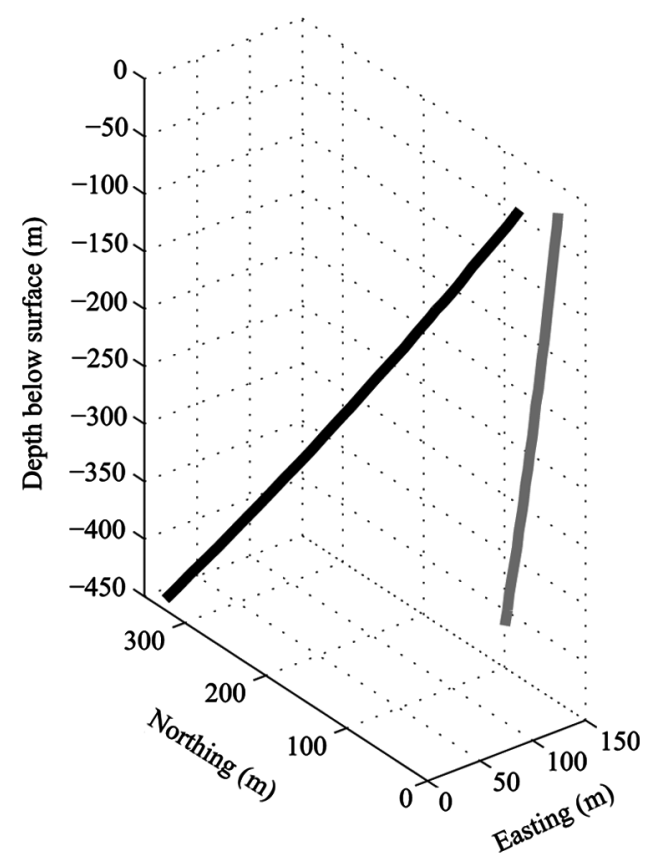

Figure 7. Relative location of two UTEM surveyed boreholes intersecting a conductor in Sudbury, Ontario, Canada (black line, $\mathrm{BH} \# 1$ and gray line, $\mathrm{BH} \# 2$ ). The roughly $800 \times 800 \mathrm{~m}$ transmitter loop is centered at approximately $900 \mathrm{~m}$ north, $50 \mathrm{~m}$ east. 
low signal values, $d B_{m} / d z_{m}$ is erratic over most of the hole due to the low S/N. As such, the apparent conductance could only be calculated over a small section of the hole where the fields were large (Figures $8 \mathrm{~d}$ and $9 \mathrm{~d}$, where $d B_{m} / d z_{m}$ is above a few $\mathrm{pT}$ ). This is not much of an issue because the fields are expected to be the largest in the areas of closest proximity to the target and that is also where the apparent conductance (equation 2) is the most reliable. If there were multiple drillholes intersecting the target, then it would be possible to image the apparent conductance variation over the targets in a similar way to the synthetic examples.

For BH\#1 and BH\#2, most apparent conductance values fall between 1000 and 10,000 S. Although the apparent conductance values do span a large range (e.g., 100-8500 S at $180 \mathrm{~m}$ for BH\#1), the apparent conductance is relatively constant over the depth range at each delay time. For instance, in $\mathrm{BH} 2$, the field values show large variations from 300- to 400-m depth across the peak in Figure $9 \mathrm{a}-9 \mathrm{c}$, but the apparent conductance values for each delay time are more or less similar across the same depth range. Because

a)

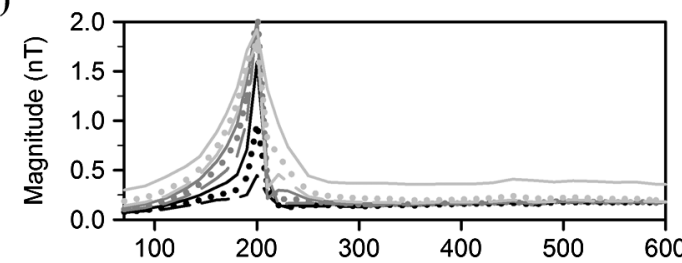

b)

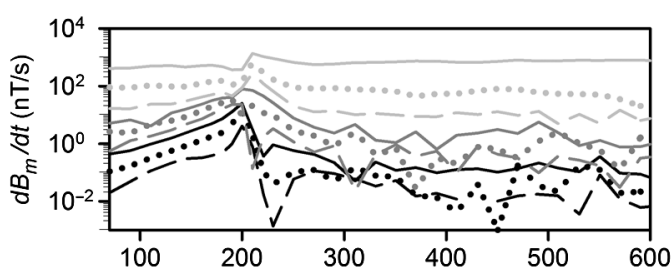

c)

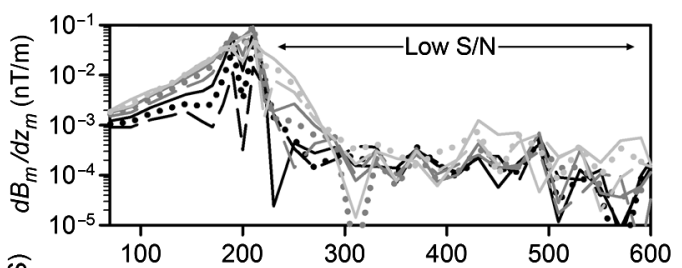

d) $\frac{\widehat{\sigma}}{8}$

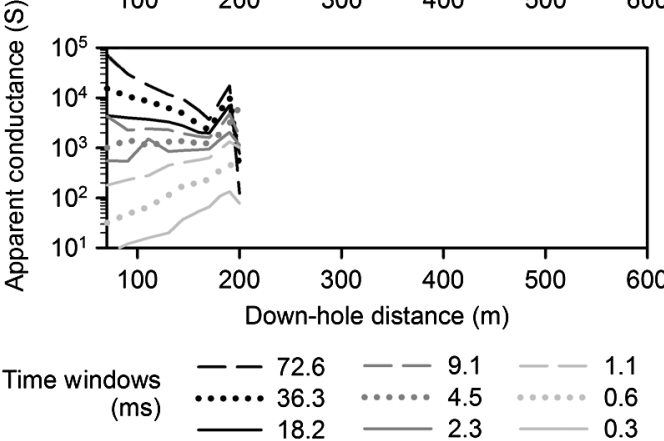

Figure 8. Results from a 4-Hz UTEM survey conducted down BH\#1 (Figure 7). (a) Magnitude of the secondary magnetic field $\left(B_{m}\right.$, theoretical primary removed). (b) Absolute magnitude of the temporal derivative $\left(d B_{m} / d t\right)$. (c) Absolute magnitude of the spatial gradient $\left(d B_{m} / d z_{m}\right)$, with the area of low S/N indicated. (d) Apparent conductance as calculated using equation 2 over the acceptable $\mathrm{S} / \mathrm{N}$ region. the results are consistent, this supports the hypothesis that the apparent conductance estimates are reliable. The increase in apparent conductance with delay time is consistent with the synthetic data, but the range is larger than what was seen in the synthetic study where the apparent conductance generally only varied within the same order of magnitude. It is possible that this discrepancy is due to the invalidation of the methods' assumptions (thin sheet, uniform conductivity distribution). Furthermore, with highly conductive bodies, the early time data represent currents that are mainly confined to the surface of the conductor and are only weakly dependent on the conductivity structure (Grant and West, 1965). With the increasing delay time, the established currents diffuse into the body and the behavior of the magnetic field will reflect the more conductive parts of the body. This is likely the reason why there is a large change in the apparent conductance from the early to the late

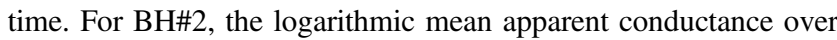
310 to $390 \mathrm{~m}$ for channels 2 to 4 (36.3 to $9.1 \mathrm{~ms}$ ) ranges from roughly 3400 to $6400 \mathrm{~S}$. For $\mathrm{BH} \# 1$, the range is slightly larger from

a)

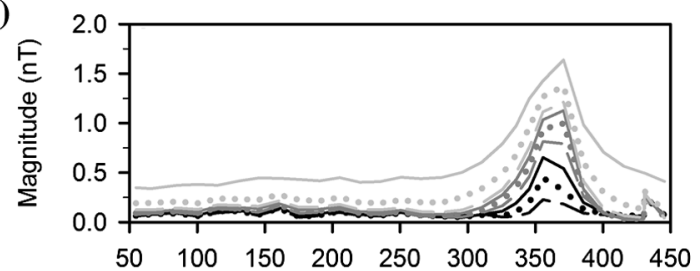

b)

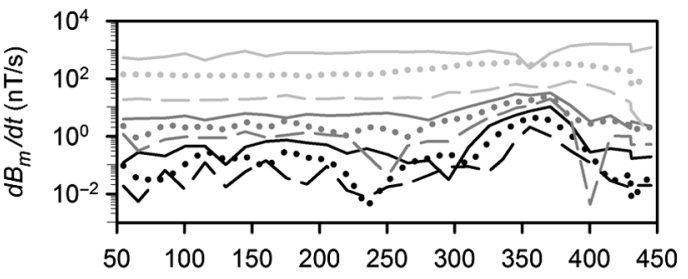

c)

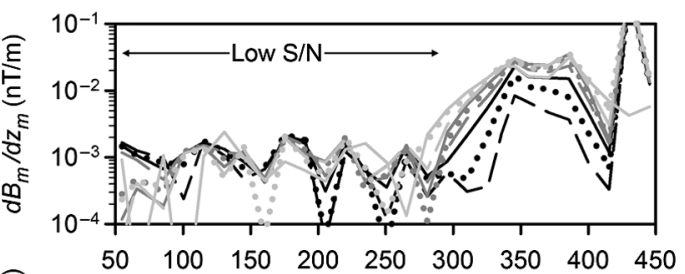

d)

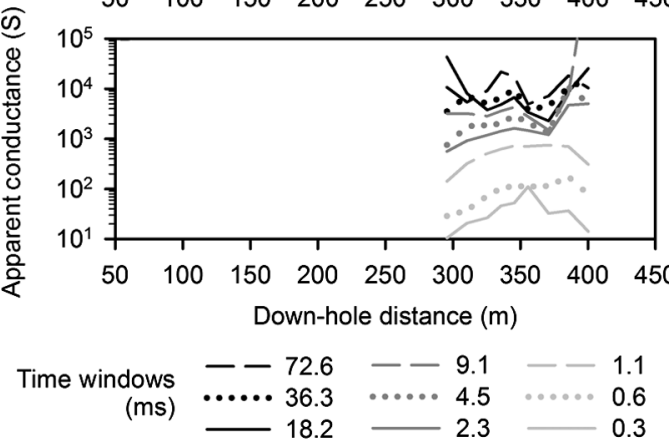

Figure 9. Results from a 4-Hz UTEM survey conducted down $\mathrm{BH} \# 2$ (Figure 7). (a) Magnitude of the magnetic field $\left(B_{m}\right)$. (b) Absolute magnitude of the temporal derivative $\left(d B_{m} / d t\right)$. (c) Absolute magnitude of the spatial gradient $\left(d B_{m} / d z_{m}\right)$, area of low S/N indicated. (d) Apparent conductance as calculated using equation 2 over the acceptable $\mathrm{S} / \mathrm{N}$ region. 
approximately 2500 to $6300 \mathrm{~S}$ for channels 2 to 4 from 70 to $190 \mathrm{~m}$. The data from $\mathrm{BH} 11$ and $\mathrm{BH \# 2}$ were also modeled in Maxwell (software by ElectroMagnetic Imaging Technology Pty. Ltd.) using conductance values of 5400 and $3000 \mathrm{~S}$, respectively (S. Dickie, personal communication, 2013), which is consistent with our results.

The conductance can also be estimated using the time constant $\tau$ of the body. The time constant was calculated analytically along the borehole (Nabighian and Macnae, 1991) using

$$
\tau=\frac{t_{2}-t_{1}}{\ln \left[B_{m}\left(t_{1}\right) / B_{m}\left(t_{2}\right)\right]},
$$

where $t_{2}$ and $t_{1}$ are adjacent time channels. The conductance of the target can then be estimated using $C \approx 10 \tau / \mu L$, where a value of $50 \mathrm{~m}$ was used for $L$ (the smallest sheet dimension). The estimate of $L$ was made by visual inspection of the narrow anomaly widths in $B_{m}$ in Figures 8 and 9. Because the time constant can be calculated at all stations and using any adjacent time channels, the conductance can be estimated along the entire hole and for each time channel pair as is done with our apparent conductance methodology (equation 2). Overall, the time constant calculated conductance ( $\tau$ conductance) produced larger conductance values at late delay times but they were generally within the same order of magnitude as our apparent conductance. The logarithmic mean $\tau$ conductance ranged from approximately 5000 to $15,000 \mathrm{~S}$ for $\mathrm{BH} \# 1$ and from 3800 to $9300 \mathrm{~S}$ to $\mathrm{BH} \# 2$ using the same delay times and stations as our apparent conductance estimate. A time constant of roughly $50 \mathrm{~ms}$ was calculated using the latest delay time at the peak amplitude in $B_{m}$ for both holes, which results in a $\tau$ conductance of roughly $8000 \mathrm{~S}$. These results are consistent with our apparent conductance estimate. However, it should be noted that estimating the conductance using the time constant can be problematic at times. The estimated time constant is only valid once the magnetic-field time dependence follows an exponential decay. This occurs at late delay times or far from the source where only the lowest order (smooth, large scale) current system dominates the measured response. At this point, the amplitudes are often small and can be greatly affected by noise. For BH\#1 and $\mathrm{BH} 2$, the estimated time constant varied substantially (10 to $100 \mathrm{~ms}$ ) between adjacent stations and delay times and was likely only valid at the peak $B_{m}$ position, where it ranged from 30 to $60 \mathrm{~ms}$ at the latest delay times. Furthermore, the estimate of the dimension $(L)$ is often highly dependent on the interpreter skill level and without forward modeling of the response the estimates can vary substantially. Although the value for $L$ is typically taken as the smallest sheet dimension, this is not always the ideal dimension. However, perhaps the two methods can be used in conjunction. For instance, if the time constant is taken as $50 \mathrm{~ms}$ and the apparent conductance is $5000 \mathrm{~S}$ (the average apparent conductance calculated using equation 2), then the dimension $L$ is calculated to be roughly $80 \mathrm{~m}$.

\section{CONCLUSION}

The conductance of an infinite uniform thin sheet can be calculated from any magnetic-field component (or combination of components) by taking the ratio of the spatial derivative in the normal direction with the temporal derivative. Through synthetic modeling, we show that by using the magnitude of the magnetic field, reliable estimates of finite-size sheets can also be obtained. This methodology is ideal for BHEM data, which typically use conductance estimation through the laborious process of forward modeling the data or through approximate conductances derived through time constant estimates. In real field data, a limiting factor is the accuracy in which the spatial gradient can be calculated with a finitedifference approach between adjacent stations because spatial gradient signals are quite low. However, we show from BHEM data acquired in a massive sulfide example in Sudbury, Ontario, Canada, that the spatial gradient is large enough in proximity to the sheet (up to $100 \mathrm{~m}$ in this example) and an apparent conductance estimate could be obtained. The estimated value is smaller at early delay times, but as large as $10,000 \mathrm{~S}$ at later delay times. The apparent conductance values are also consistent with the Maxwell models. Calculating the target's apparent conductance from a simple ratio derived from BHEM data is an attractive complement to (1) logging the hole with a conductivity probe, (2) traditional inversions, forward modeling, and time-constant-derived conductance values, and (3) using handheld instruments or laboratory techniques on the recovered core. Furthermore, the field data suggest that the apparent conductance can be used in conjunction with a calculated time constant to estimate the dimensions of the target. Future research will investigate this possibility further.

\section{ACKNOWLEDGEMENTS}

We are grateful to the following for financial support of this research: NSERC, Vale, Sudbury Integrated Nickel Operations, a Glencore Company, Wallbridge Mining, KGHM International, and the Centre for Excellence in Mining Innovation (CEMI). We are thankful to B. Polzer for suggesting that our previous work could be applied to BHEM data and for suggestions and comments that improved this paper. We are also grateful to Vale, particularly G. McDowell and S. Dickie, for providing the borehole field data and for helpful comments and suggestions. M. Kolaj is grateful for an NSERC Alexander Graham Bell scholarship, an SEG George V. Keller Scholarship, and an SEG/KEGS Ontario Scholarship. MultiLoop III was provided by Lamontagne Geophysics, and Peter Walker guided us in its use. We are also grateful to J. Macnae for his suggestion to include a discussion on conductance estimates using the estimated time constant of the target.

\section{REFERENCES}

Dyck, A. V., 1991, Drill-hole electromagnetic methods, in M. N. Nabighian, ed., Electromagnetic methods in applied geophysics, vol. 2: Applications: Parts A and B: SEG, Investigations in Geophysics 3, 881-930.

Grant, F. S., and G. F. West, 1965, Interpretation theory in applied geophysics: McGraw-Hill.

King, A., 2007, Review of geophysical technology for Ni-Cu-PGE deposits, in B. Milkereit, ed., Proceedings of Exploration 07: Fifth Decennial International Conference on Mineral Exploration, 647-665.

Kolaj, M., and R. S. Smith, 2013, Using spatial derivatives of electromagnetic data to map lateral conductance variations in thin sheet models: Applications over mine tailings ponds: Geophysics, 78, no. 5, E225-E235, doi: 10.1190/geo2012-0457.1.

Lamontagne, Y., 2007, Deep exploration with EM in boreholes, in B. Milkereit, ed., Proceedings of Exploration 07: Fifth Decennial International Conference on Mineral Exploration, 401-415.

Lelièvre, P. G., D. W. Oldenburg, and N. C. Williams, 2009, Integrating geological and geophysical data through advanced constrained inversions: Exploration Geophysics, 40, 334-341, doi: 10.1071/EG09012.

Li, Y., and D. W. Oldenburg, 2000, Incorporating geological dip information into geophysical inversions: Geophysics, 65, 148-157, doi: 10.1190/1 .1444705 .

McDowell, G., A. Mackie, and M. Palkovits, 2007, Grade estimation at CVRD Inco's Canadian sulphide mines: Symposium on the Application 
of Geophysics to Engineering and Environmental Problems, SEG, 1103-1112, doi: $10.4133 / 1.2924616$.

Nabighian, M. N., and J. C. Macnae, 1991, Time domain electromagnetic prospecting methods, in M. N. Nabighian, ed., Electromagnetic methods in applied geophysics, vol. 2: Applications: Parts A and B: SEG, Investigations in Geophysics 3, 427-520.

Oldenburg, D. W., and D. A. Pratt, 2007, Deep exploration with EM in boreholes, in B. Milkereit, ed., Proceedings of Exploration 07: Fifth Decennial International Conference on Mineral Exploration, 61-95.

Palacky, G. J., 1987, Resistivity characteristics of geological targets, in M. N. Nabighian, ed., Electromagnetic methods in applied geophysics, vol. 1: Theory: Parts A and B: SEG, Investigations in Geophysics, 53-125.

Polzer, B., 2000, The role of BHEM in the discovery and definition of the Kelly Lake Ni-Cu Deposit, Sudbury, Canada: 56th Annual International Meeting, SEG, Expanded Abstracts, 1063-1066.

Price, A. T., 1949, The induction of electric currents in non uniform thin sheets and shells: Quarterly Journal of Mechanics and Applied Mathematics, 2, 283-310, doi: 10.1093/qjmam/2.3.283.

Smith, R. S., and A. P. Annan, 2000, Using an induction coil sensor to indirectly measure the B-field response in the bandwidth of the transient electromagnetic method: Geophysics, 65, 1489-1494, doi: 10.1190/1 1444837 .

Smith, R., M. Shore, and D. Rainsford, 2012, How to make better use of physical properties in mineral exploration: The exploration site measurement: The Leading Edge, 31, 330-337, doi: 10.1190/1.3694901.

Smith, R. S., and G. F. West, 1987, Electromagnetic induction in an inhomogeneous conductive thin sheet: Geophysics, 52, 1677-1688, doi: 10 $1190 / 1.1442284$.

Telford, W. M., L. P. Geldart, and R. E. Sheriff, 1990, Applied geophysics: Cambridge University Press.

Walker, P., and Y. Lamontagne, 2006, Electromagnetic interpretation in complex geological environments: 76th Annual International Meeting, SEG, Expanded Abstracts, 1288-1292.

West, G. F., J. C. Macnae, and Y. Lamontagne, 1984, A time-domain EM system measuring the step response of the ground: Geophysics, 49, 10101026, doi: 10.1190/1.1441716.

Zhang, Z., and J. Xiao, 2001, Inversions of surface and borehole data from large-loop transient electromagnetic system over a 1-D earth: Geophysics, 66, 1090-1096, doi: 10.1190/1.1487056. 